\title{
Analysis of the Performances of Sealed Timing Resistive Plate Chambers (tRPCs)
}

\author{
Khokon Hossen* \\ Max-Planck Institute for Nuclear Physics, Heidelberg, Germany \\ *Corresponding author: hossen@mpi-hd.mpg.de \\ Received June 17, 2014; Revised July 02, 2014; Accepted July 29, 2014

\begin{abstract}
Resistive Plate Chambers (RPCs) were introduced by R. Santonico and R. Cardarelli in 1981, are gas ionization chambers made with resistive electrodes separated by precision spacers. Typical gas gap ranges from a few hundred micrometers to several millimeters wide. Timing Resistive Plate Chambers (tRPCs) were introduced in 2000 by P.Fonte, A. Smirnitsky and M.C.S Williams and since then it has reached Time Resolutions better than 50 ps $(\sigma)$ with efficiencies above 99\% for Minimum Ionizing Particle (MIP). In this research paper, we described the main features of gas detectors and the different types of RPCs and their properties. We described the main results that we have got during the operating the sealed tRPCs built in the laboratory.
\end{abstract}

Keywords: RPCs, tRPCs, gas ionization chambers, efficiency, performances

Cite This Article: Khokon Hossen, “Analysis of the Performances of Sealed Timing Resistive Plate Chambers (tRPCs).” International Journal of Physics, vol. 2, no. 4 (2014): 105-108. doi: 10.12691/ijp-2-4-2.

\section{Introduction}

Resistive Plate Chambers (RPCs) and Timing Resistive Plate Chambers (tRPCs) belongs to the family of the gas ionization detector. The first RPC was introduced by R. Santonico and R. Cardarelli in 1981 and tRPCs were introduced by P.Fonte, A. Smirnitsky and M.C.S Williams in 2000 [1]. Since then, Resistive Plate Chambers (RPCs) have become very popular detectors to cover very big surface because they offer a very high time resolution at a very good performance and also price ratio compared with other techniques [2]. Nowadays, many experiments in Nuclear Physics, High Energy Physics, Medical Physics, Particle Physics and Astrophysics are using RPCs [3].

Such types of detectors could also be very helpful for small experimental setups as an alternative to other methods. But they have the disadvantage of needing very bulky and uncomfortable gas systems [4]. For this reason, in this research, we have developed a cheap and easy to build small and portable sealed tRPCs. We have also analyzed some of their performances and behaviors.

\section{Setup and the Acquisition System}

We have tested whether our sealed tRPCs work properly or not before took data.

Figure 1 shows the block diagram of the acquisition setup that we used to test our sealed tRPCs and getting data. We took data using ${ }^{22} \mathrm{Na}$ source to produce the signal and to analyze the performances of our detector. We used a fast scintillator, readout by two photomultipliers, to make coincidences and rejecting the electrical noise.

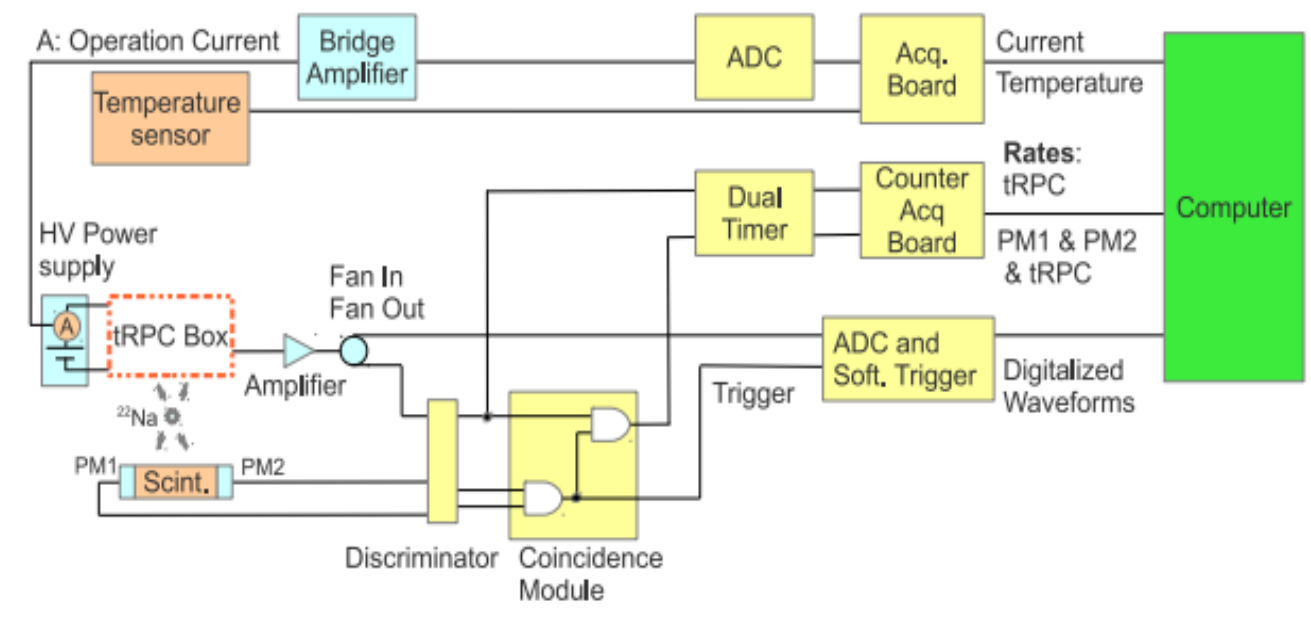

Figure 1. Block Diagram of Acquisition setup 
The ${ }^{22} \mathrm{Na}$ source emits positrons $\left(\mathrm{e}^{+}\right)$. When a positron annihilates with an electron of the matter almost at rest, it produced two gammas of energy $\approx 0.511 \mathrm{Mev}$, each one travelling in opposite directions [5]. If we put the ${ }^{22} \mathrm{Na}$ source between the detector and the scintillator, we can get a clean sample of gammas hitting the detector, making trigger on the scintillator. The typical reaction is

$$
\begin{aligned}
& { }^{22} \mathrm{Na} \rightarrow{ }^{22} \mathrm{Ne}^{*}+\mathrm{e}^{+}+v \\
& \mathrm{e}^{+}+\mathrm{e}^{-} \rightarrow 2 \gamma_{0.511 \mathrm{MeV}}
\end{aligned}
$$

If the current intensity shows around 0.2 or $0.3 \mathrm{nA} / \mathrm{cm}^{2}$ with the increase of voltage up to 3000 Volts then we can consider that our sealed tRPCs is ready for taking data.

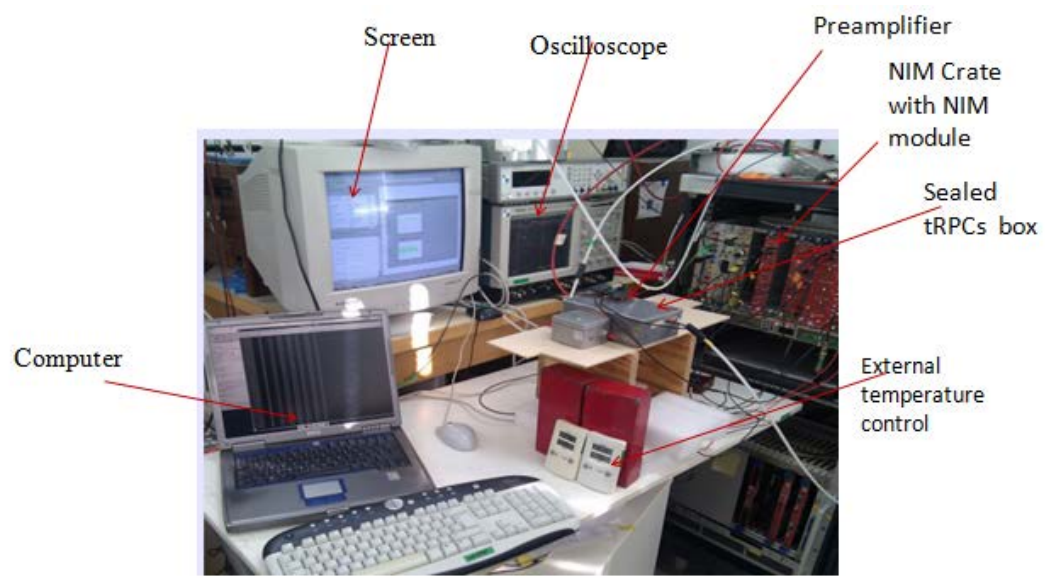

Figure 2. Experimental arrangement of testing and getting data of tRPCs

Figure 2 shows the experimental arrangement of testing and getting data of sealed tRPCs. The indicator shows the tRPCs Box, Gas tube, Computer, Oscilloscope and Electronics plate etc.

\subsection{Commissioning}

Once the chamber is finished, we put it in Freon gas during $\sim 24$ hours in order to remove any rest of other gases in the circuit [6]. Then we put it in gas and we increase the HV step by step slowly controlling that the experiment of the HV source stay below $\sim \mathrm{nA}$ until reaching the normal working potentials between $2800 \mathrm{~V}$ and $7000 \mathrm{~V}$.

\section{Results}

At the last step of our work, we have taken data for the analysis of the behaviors of our detector. All the data were taken with the oscilloscope using the acquisition setup described in the previous section. All the information of the digitalized waveforms provided by the oscilloscope, time and voltage, where stored in files and the analysis was done off-line. Time is given in ns and voltage is given in $\mathrm{mV}$. For this analysis, we used Root Program [7].

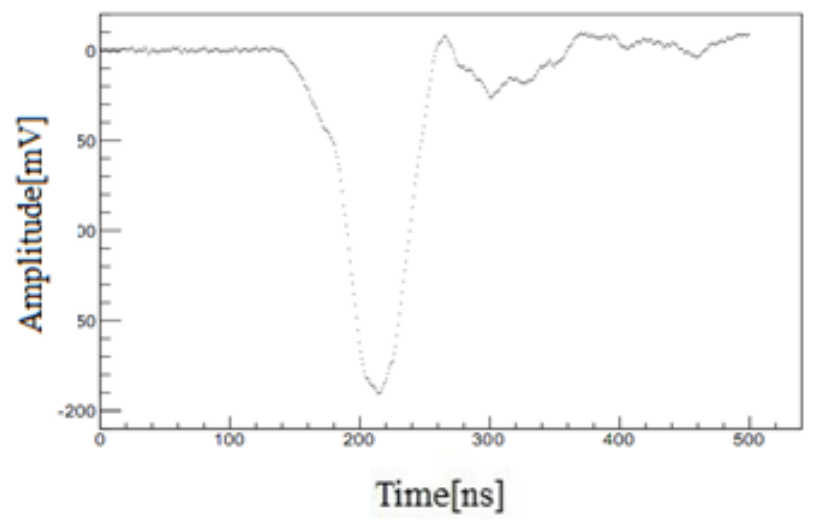

Figure 3. Example of a good signal/event
Figure 3 shows a typical good pulse. The noise background before the pulse is non-significant, showing a good electrical behavior. The irregular shape of the waveform after the pulse is an effect of the shaping circuit.

Figure 4 shows an example of two almost simultaneous avalanches producing a bad event with a wrong charge and amplitude measurements.

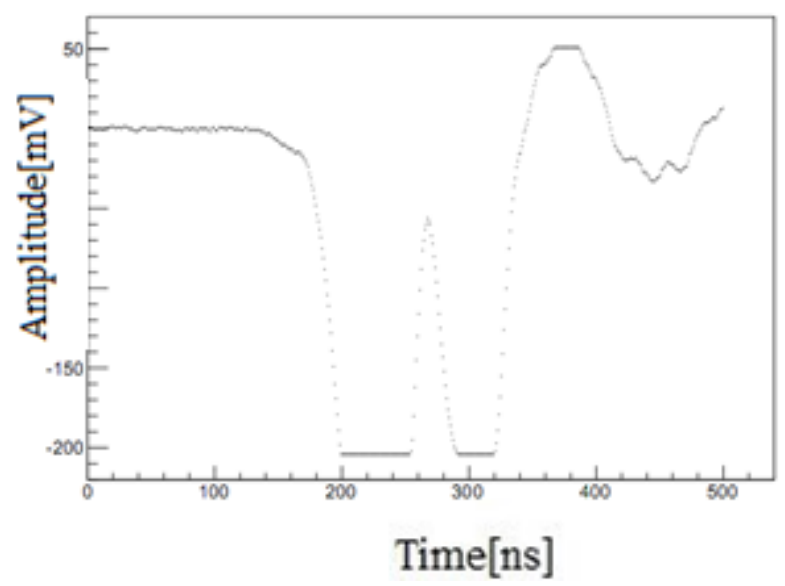

Figure 4. Example of a bad signal/event

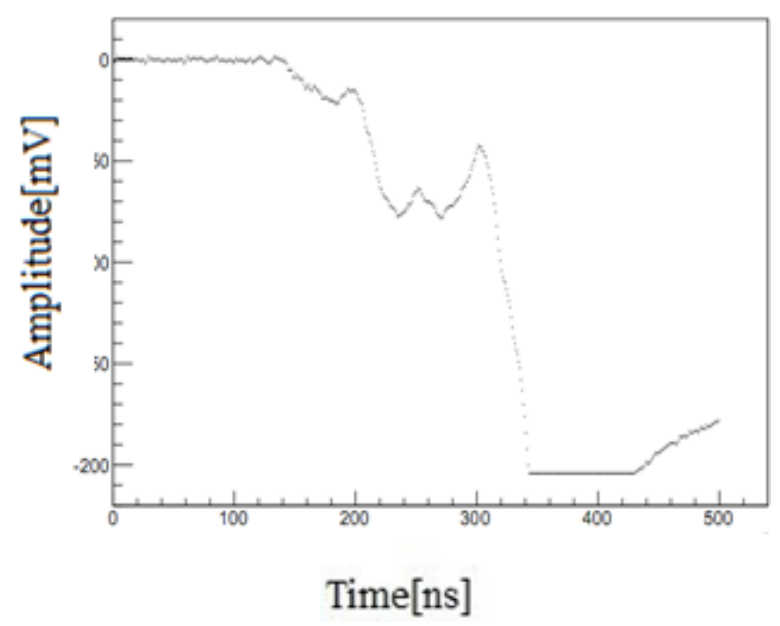

Figure 5. An example of Streamer formation 
Figure 5 shows a typical streamer event that saturated the oscilloscope both in amplitude and in time. The small peak before the maximum is known as the precursor of the streamer. The charge was calculated integrating the signal and the amplitude was calculated looking for the maximum of the waveform (Figure 6, Figure 7, Figure 8).

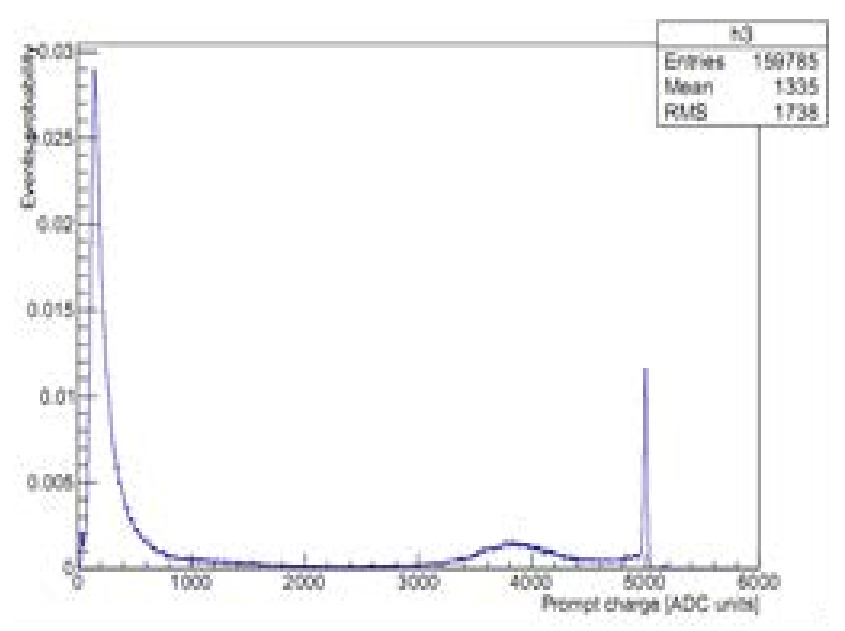

Figure 6. Total Prompt charge spectrum for maximum charge

The Figure 6 shows the total Prompt charge spectrum for all the recorded events. The first peak at around point 200[ADC units] of the spectrum should correspond to gammas from the ${ }^{22} \mathrm{Na}$ source that come into the sealed tRPCs. The second peak at the charge around 4000[ADC units] could correspond to either to big charge bad events, like the one shown in Figure 4, or to streamer events. The peak at 5000 corresponds to charges above 5000 units. The very small peak at the left of the histogram corresponds to the pedestal produced by low charge noise events.

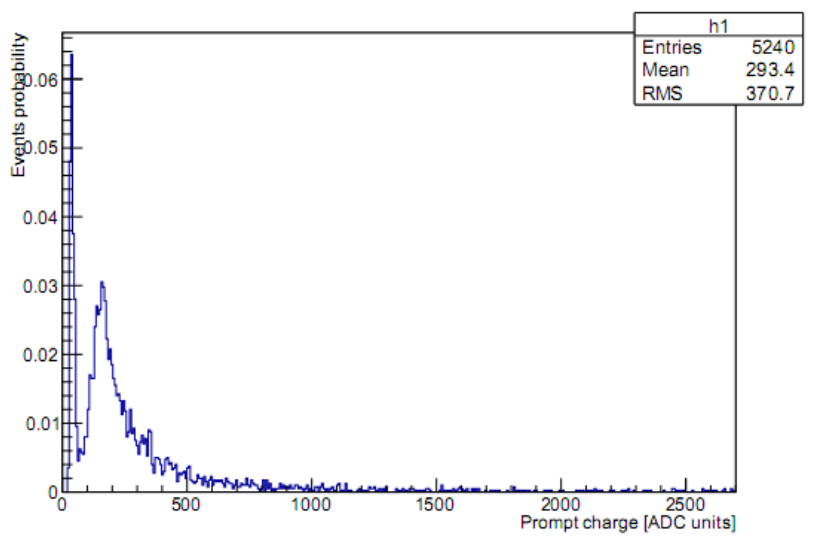

Figure 7. Prompt charge spectrum for charge as it is coincidence

The Figure 7 shows the Prompt charge spectrum for all the events in coincidence with the scintillator. The peak at charge 200 [ADC units] corresponds to a gamma produced in opposite direction that the one that triggered the coincidence in the scintillator. The big pedestal shows that our threshold level for rejecting noise was quite low and that some noise as in coincidence with the gamma at the scintillator.

The Figure 8 shows the amplitude spectrum for all the events in coincidence with the scintillator. The peak at around $20 \mathrm{mV}$ corresponds to the gammas and some of the pedestal events shown in the Figure 7. The big peak at the right side correspond to events that reached the maximum amplitude accepted by the oscilloscope. Figure 9 shows the Amplitude vs Charge plot for all events. It shows that there is a linear relationship between the amplitude and the charge as it was expected. We also observe that some of the gammas, saturated signal and steamers reached the maximum amplitude as it were already commented previously. The bottom left small structure correspond to noise pedestal events.

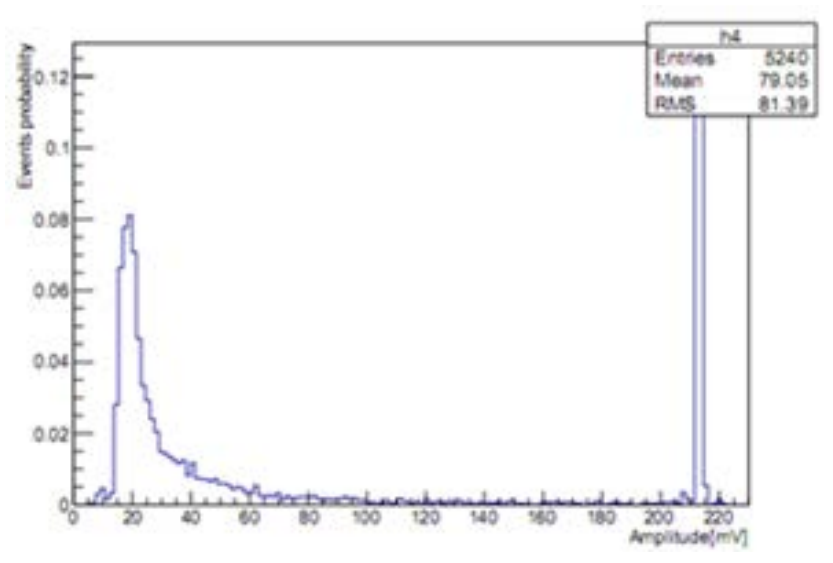

Figure 8. Amplitude distribution in tRPCs

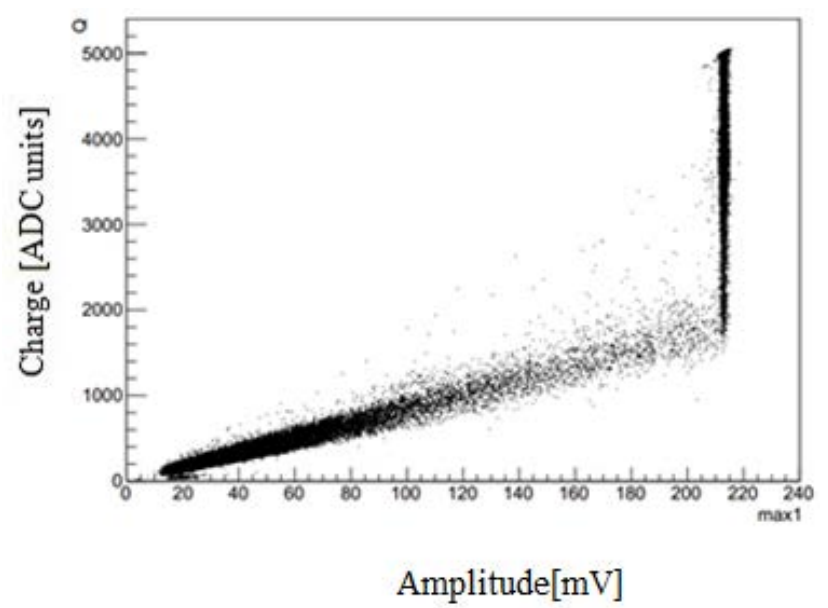

Figure 9. Amplitude and Charge distribution in tRPCs

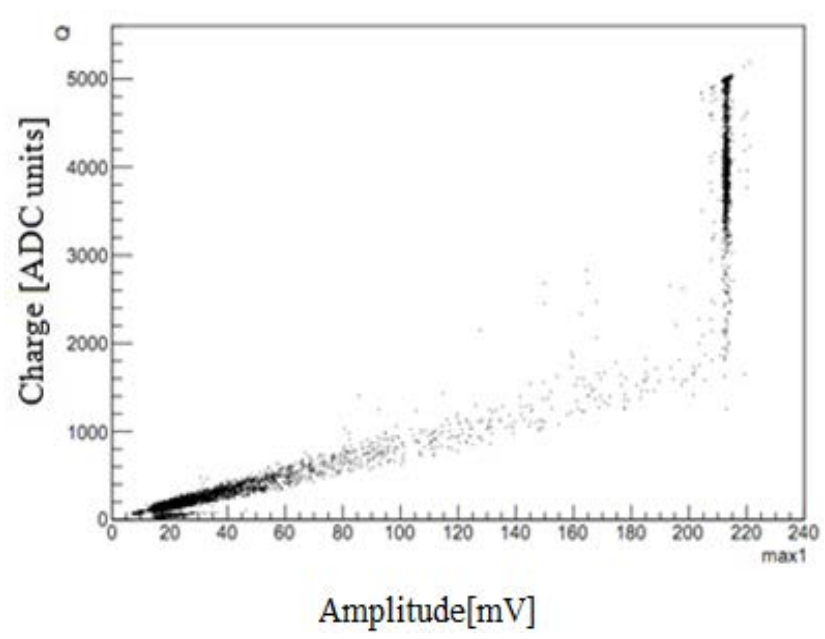

Figure 10. Amplitude and Charge distribution in tRPCs (Coincidence)

The Figure 10 shows the Amplitude vs Charge plot only for coincidence events. All the good events, the pedestal noise events and the high charge bad events can be easily seen. 
Figure 11 shows the slope spectrum for the coincidence events. It shows three structures that would need further analysis. Perhaps they are related with the number of primary electrons in the avalanche. The Figure 12 shows the time difference between two sides of the detector. It shows a good behavior.

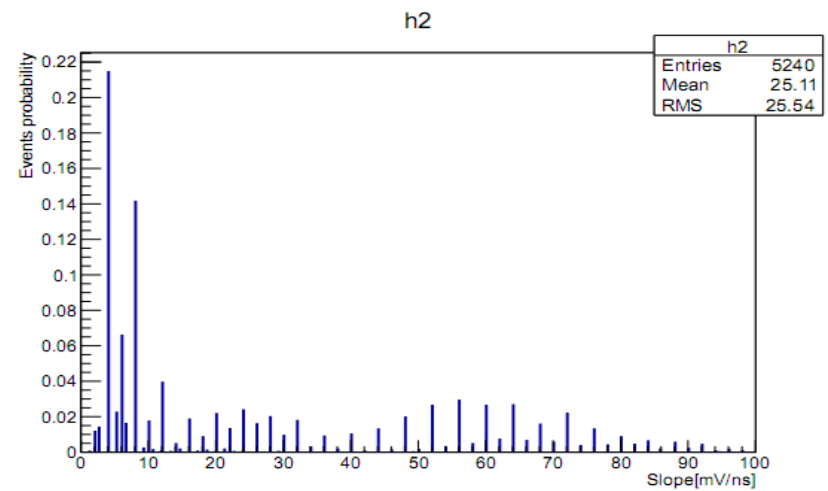

Figure 11. Slope distribution in tRPCs (coincidence)

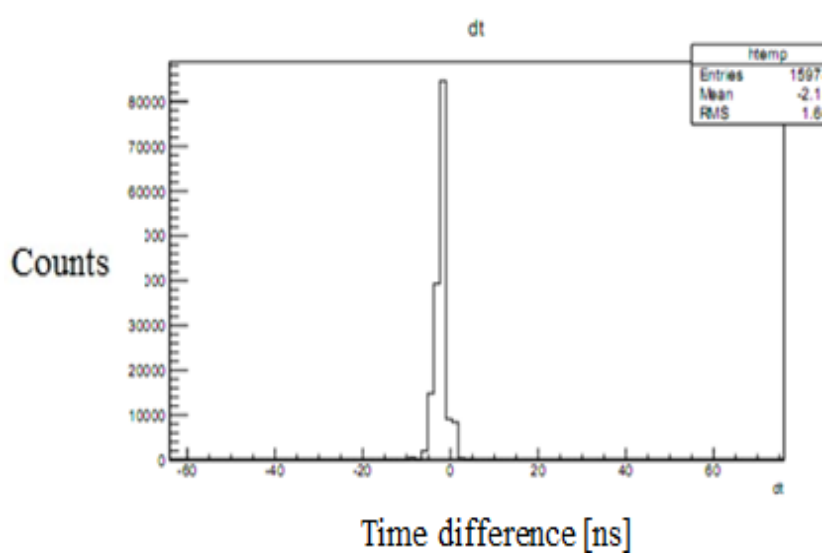

Figure 12. Time difference between two sides in tRPCs

\section{Conclusions}

In this research paper, we have explained the performances of timing Resistive Plate Chambers (tRPCs).

We have built several low consuming sealed tRPCs and explain the method of building tRPCs with way common and cheap materials. We have commissioned one detector in the laboratory and make it work. We have operated the detector and we have taken data with an experimental setup allowing making coincidence with a fast scintillator detector. We have analised the data showing that the detector works properly with a very low electrical noise and producing significative pulses both with gammas and cosmics. We have mainly analised the charge and the amplitude of the events both in coincidence and without the coincidence with the scintillator. The detector keeps ready for further studies.

\section{References}

[1] P.Fonte, A. Smirnitsky and M.C.S Williams, NIM, A 443, (2000), 201

[2] Oxford Physics, C4: Particle Physics Major Option Particle Detectors

[3] William R. Leo, Techniques for Nuclear and Particle Physics Experiments.

[4] http://consult.cern.ch/writeup/magboltz/.

[5] P. Fonte, V. Peskov, B.D. Ramsey, "Streamers in MSGC's and other gaseous detectors" $1-13$

[6] Nicholas Tsoulfanidis, Measurement and Detection of Radiation, Second Edition.

[7] http://root.cern.ch/drupal/. 